\title{
Inheritance of a Novel COL8A2 Mutation Defines a Distinct Early-Onset Subtype of Fuchs Corneal Dystrophy
}

\author{
Jobn D. Gottsch, ${ }^{1}$ Olof H. Sundin, ${ }^{1,2,3}$ Sammy H. Liu, ${ }^{1}$ Albert S. Jun, ${ }^{1}$ Karl W. Broman, ${ }^{4}$ \\ Walter J. Stark, ${ }^{1}$ Elizabeth C. L. Vito, ${ }^{1}$ Amol K. Narang, ${ }^{1}$ John M. Thompson, ${ }^{1}$ and \\ Malcolm Magovern ${ }^{1}$
}

Purpose. To characterize the genetic basis and phenotype of inherited Fuchs corneal dystrophy (FCD).

Methods. DNA from blood was used for genome-wide linkage scans with tandem repeat polymorphisms. Mutation detection involved sequencing PCR-amplified exons. Families with FCD were clinically evaluated and graded on the Krachmer severity scale. Confocal specular microscopy visualized the morphology of endothelial guttae, small protrusions of Descemet's membrane that are characteristic of FCD.

Results. Linkage was obtained to 1p34.3-p32 for the autosomal dominant kindred originally reported by Magovern in 1979. All 21 cases with FCD and one with posterior polymorphous dystrophy were heterozygous for L450W, a novel point mutation in the COL8A2 gene. Of 62 independent cases of familial FCD, none had the previously reported mutations in COL8A2. Corneal guttae in COL8A2 patients were small, rounded, and associated with the endothelial cell center. This contrasts with common FCD, in which guttae were larger, sharply peaked, and initially positioned at edges of endothelial cells. The profile of age and disease severity for the L450W FCD kindred suggested that disease onset occurred in infancy, compared with an average age of onset of 50 years estimated for 201 familial FCD patients in 62 other families.

Conclusions. A novel pathogenic L450W COL8A2 mutation was identified and its highly distinctive pathology characterized. This indicates that COL8A2 mutations give rise to a rare subtype of FCD. This study also provides the first direct evidence that $C O L 8 A 2-\mathrm{FCD}$ progresses from early to late stages in 25 years, a rate similar to that estimated for late-onset FCD. (Invest Ophthalmol Vis Sci. 2005;46:1934-1939) DOI: 10.1167/iovs.04-0937

From the ${ }^{1}$ Center for Corneal Genetics, Cornea and External Disease Service, the ${ }^{2}$ Laboratory of Developmental Genetics, The Wilmer Eye Institute, Johns Hopkins University School of Medicine, Baltimore, Maryland; and the ${ }^{3}$ Department of Molecular Biology and Genetics, and the ${ }^{4}$ Department of Biostatistics, Johns Hopkins Bloomberg School of Public Health, Baltimore, Maryland.

Supported by The Josephine S. Sutland and the Shelia S. and Dr. Lawrence C. Pakula Research Fund, and The Commonwealth Research Fund.

Submitted for publication August 3, 2004; revised November 5 , 2004; accepted December 10, 2004.

Disclosure: J.D. Gottsch, None; O.H. Sundin, None; S.H. Liu, None; A.S. Jun, None; K.W. Broman, None; W.J. Stark, None; E.C.L. Vito, None; A.K. Narang, None; J.M. Thompson, None; M. Magovern, None

The publication costs of this article were defrayed in part by page charge payment. This article must therefore be marked "advertisement" in accordance with 18 U.S.C. $\$ 1734$ solely to indicate this fact.

Corresponding author: John D. Gottsch, The Wilmer Eye Institute, Johns Hopkins Hospital, 600 N. Wolfe Street, Baltimore, MD 21287; jgottsch@jhmi.edu.
Guchs corneal dystrophy (FCD) is a primary disorder of the endothelium that leads to progressive edema of the corneal stroma (OMIM 136800; Online Mendelian Inheritance in Man; http://www.ncbi.nlm.nih.gov/Omim/ provided in the public domain by the National Center for Biotechnology Information, Bethesda, MD). Visual disability from this disease is currently the major reason for corneal transplantation. ${ }^{1}$ The initial stages of FCD typically begin in the fifth through seventh decades of life and are characterized by localized thickening of Descemet's membrane and the development of nodular excrescences called guttae. This early phase is followed by long-term decreases in the density and ion transport functions of the overlying corneal endothelial cells, which allows excess water to accumulate in the cornea. ${ }^{2-8}$ Several reports, including the original description by Fuchs, have indicated that two to three times as many females as males are affected by the disease. ${ }^{2,7,9}$

As many as $50 \%$ of clinical cases of FCD may show familial clustering, ${ }^{9}$ and the disease generally follows an autosomal dominant pattern of inheritance.$^{10-15}$ Nearly all these families show inheritance of late-onset FCD, whereas rare cases ${ }^{12,14}$ show disease onset as early as the first decade, with extensive corneal edema by the third or fourth decades. Recently, a multigenerational family exhibiting advanced FCD by their third and fourth decades revealed genetic linkage to a $7 \mathrm{cM}$ region of chromosome 1p34.3-p32. ${ }^{14}$ Individuals affected with FCD were found to be heterozygous for a point mutation altering the collagen helix domain of the $\alpha 2$ chain of type VIII collagen (COL8A2), substituting a lysine for a glutamine (Q455K). Because collagen VIII is a major component of Descemet's membrane, the mutation provides important evidence that the disease originates as a molecular dysfunction of the extracellular matrix of the endothelium. A second independently ascertained, large family, also described as having early onset, had exactly the same Q455K mutation. ${ }^{14}$ This second family showed perfect concordance between the mutation and individuals affected with FCD. In a third independent small family, ${ }^{14}$ the $\mathrm{Q} 455 \mathrm{~K}$ mutation was associated with two patients with posterior polymorphous corneal dystrophy (PPCD), a distinct endothelial cell disorder with some features similar to FCD. To date, no COL8A2 amino acid sequence variants have been identified that are clearly associated with late-onset FCD. ${ }^{14,16}$

We have performed genetic linkage analysis of the large early-onset FCD family originally described by Magovern et al., ${ }^{12}$ and identified a second COL8A2 mutation, L450W. We report that this mutation is associated with highly distinctive guttae that differ from those of common late-onset FCD and suggest that mutations in $C O L 8 A 2$ give rise to a rare subtype of FCD. Long-term study of this family has also provided us with the unique opportunity to follow the temporal progression of inherited FCD.

\section{Materials ANd Methods}

\section{Families}

A total of 63 families were recruited after initial evaluations of patients with FCD at the Cornea Service of the Wilmer Ophthalmological 


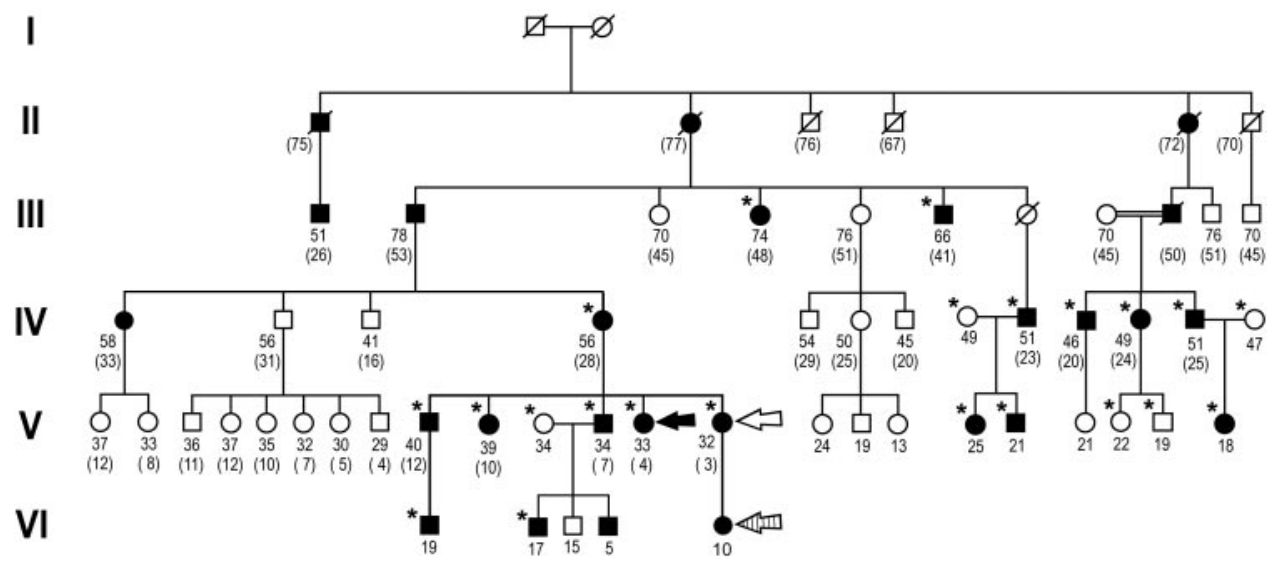

Figure 1. Pedigree as described by Magovern et al. ${ }^{12}$ in 1979 with additions in 2003. Squares: males. Circles: females. Diagonal lines: currently deceased. Asterisk: the patient was genotyped and subjected to linkage analysis. A number without parentheses is the individual's age when examined in 2003 for the present study, and indicates DNA was collected. Number in parentheses indicates age of the individual when examined for the 1979 study. Filled symbols: clear diagnosis of Fuchs corneal dystrophy; open symbols with age shown: normal diagnosis; open symbols without age: subject not examined and therefore is of unknown phenotype. Filled and open arrows: patients with FCD appearing in Figures 2 and 3. Hatched arrow: patient with PPCD shown in Figure 4.

Institute. The study protocol was approved by the Institutional Review Board for Human Subjects Research at the Johns Hopkins University School of Medicine and was in accordance with the tenets of the Declaration of Helsinki. Written informed consent was obtained from all study participants.

\section{Severity Grading, Photography, and Confocal Specular Microscopy}

Patients were photographed to demonstrate the area and number of confluent guttae by direct, indirect, and retroillumination. Grading was a modification of the scale proposed by Krachmer et al. ${ }^{9}$ Grade 0 indicated no disease, or an inconclusive finding, with 0 to 11 central guttae; grade 1, definitive onset of the disease, indicated by 12 or more central, nonconfluent guttae in at least one eye; grade 2, a zone of confluent central guttae 1 to $2 \mathrm{~mm}$ in width; grade 3 , a greater than 2 to 5-mm wide zone of confluent guttae in the central cornea; grade 4 , a central region of confluent guttae greater than $5 \mathrm{~mm}$ in width; and grade 5 , the same as grade 4 , but with added disease involving stromal or epithelial edema. Confocal specular microscopy $5,6,17$ was performed (ConfoScan3; Nidek Technologies, Vigonza, Italy), to provide digital imaging of guttae in Descemet's membrane and to obtain an en face image of endothelial cells.

\section{Genotyping and Linkage Analysis}

Venous blood $(10 \mathrm{~mL})$ was collected and frozen at $-20^{\circ} \mathrm{C}$. DNA was extracted using standard methods (Qiagen, Santa Clara, CA). Genotyping of polymorphic linkage markers was performed by the University of Utah Genomics Core Facility (Salt Lake City, UT; http://www. cores.utah.edu/genomics/index.htm). Genome-wide scans were performed with fluorescently labeled primers of the MD10 microsatellite marker set (Applied Biosystems Inc. [ABI], Foster City, CA). Standard PCR conditions (ABI) were used, and products were ethanol precipitated and electrophoresed through 5\% polyacrylamide gels on an automated DNA sequencer (Prism 377; ABI). Initial data analysis was performed on computer (GeneScan 3.1 and Genotyper 2.1 software; $\mathrm{ABI}$. RelCheck ${ }^{18,19}$ was used to verify the relationships in the pedigree. Pedcheck ${ }^{20}$ was used in the identification and resolution of Mendelian inconsistencies in the genotype data. Marker allele frequencies were estimated by counting alleles in the observed individuals, ignoring familial relationships. Although the resultant estimates were not ideal, they are unbiased, ${ }^{21}$ and the results of multipoint linkage analysis are largely unaffected by these values. SimWalk 2 version 2.89 was used to perform multipoint parametric linkage analysis and to infer haplotypes, ${ }^{22}$ under the assumption of a dominant, completely penetrant locus, with disease allele frequency $10^{-4}$. The order of genetic markers and intermarker distances were taken from the Marshfield maps. $^{23}$

\section{Mutation Screening of $C O L 8 A 2$}

Genomic DNA was purified as above and exons of the COL8A2 gene PCR amplified ${ }^{24}$ with the 16 sets of primer pairs used in earlier work. ${ }^{14}$ The PCRs were performed with the GC-genomic polymerase system (BD Biosciences, Palo Alto, CA) in a total volume of $50 \mu \mathrm{L}$ using $100 \mathrm{ng}$ of each genomic DNA samples as templates. GC melt (final concentration of $1.0 \mathrm{M}$ ) was added to PCR reactions to alleviate secondarystructure problems created by the very high GC content of COL8A2. The PCR conditions were as follows: initial denaturation $\left(95^{\circ} \mathrm{C}, 1\right.$ minute) followed by 35 cycles of denaturation $\left(94^{\circ} \mathrm{C}, 30\right.$ seconds), annealing $\left(62.0^{\circ} \mathrm{C}\right.$ for primer 12,30 seconds), extension $\left(72^{\circ} \mathrm{C}, 90\right.$ seconds), and final extension $\left(72^{\circ} \mathrm{C}, 5\right.$ minutes). PCR products were purified (QIAquick columns; Qiagen) and directly analyzed on an automated DNA sequencer (Prism 377; ABI). Nucleotide sequences were compared with the published cDNA sequence of the COL8A2 gene by BLAST search.

\section{Age, Sex, and Severity of Disease in Families with FCD}

Families with and without COL8A2 mutations were compared as to age, sex, and severity of disease. The statistical significance of the observed earlier onset of Fuchs in the Magovern pedigree was assessed by comparing the average age of individuals with severity of grade 3 or greater in this pedigree versus other pedigrees by a permutation test ${ }^{25}$ with 1,000,000 permutation replicates. The statistical significance of the difference in sex ratios among affected individuals (with severity $\geq 1$ ) in the Magovern pedigree versus other pedigrees was assessed by the Fisher exact test.

\section{Results}

\section{Genetic Linkage and Mutation Screen}

The pedigree (Fig. 1) was analyzed by testing 17 affected and 5 unaffected family members with microsatellite markers. These covered all 22 autosomes, with an average spacing of 10 centimorgans (cM). Multipoint linkage analysis identified a 22-Mb disease interval between D1S234 and D1S2797 on 

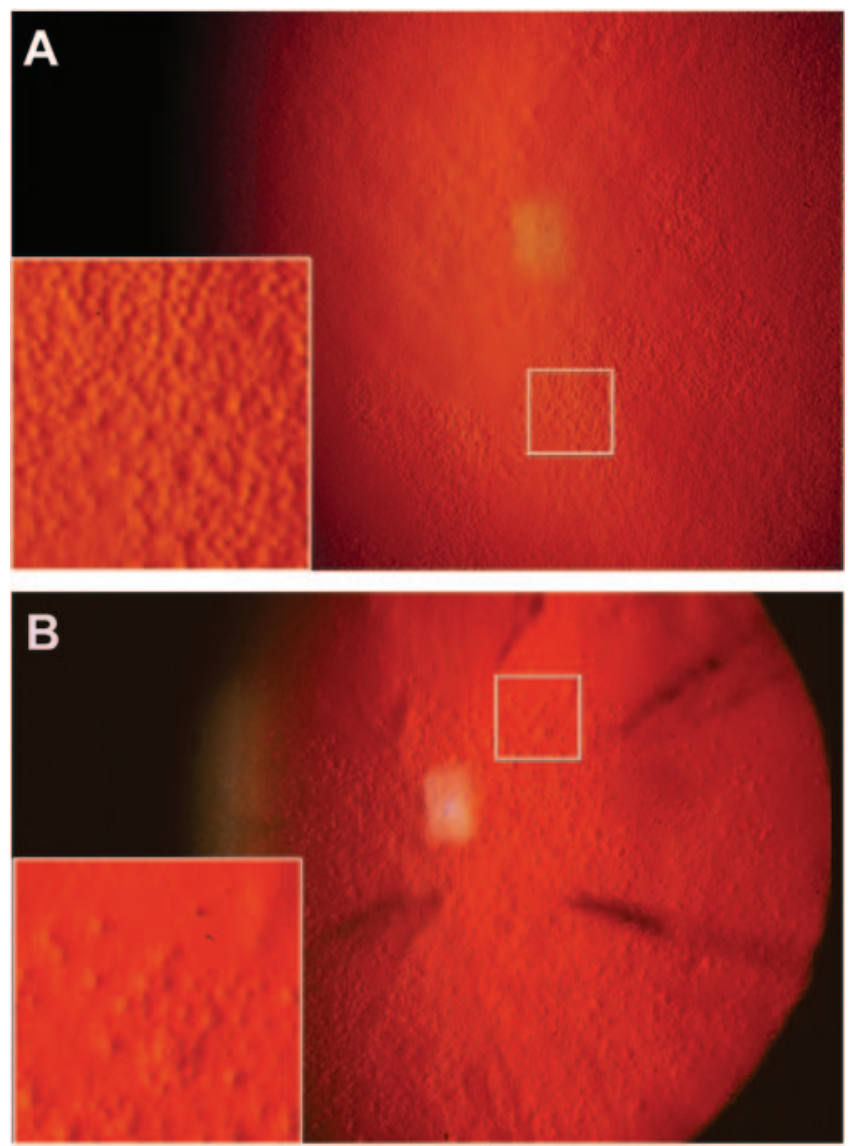

FIGURE 2. (A) Retroillumination image of a patient with disease of grade 3 severity (age 33 ) with the L450W-COL8A2 mutation (Fig. 1, filled arrow). The cornea revealed large affected patches, with a fine-grained distribution of guttae. Inset: $3.8 \times$ enlargement of a representative affected area (rectangle) with densely packed low-elevation guttae. (B) Similar views of a patient with late-onset grade 3 FCD with no known mutations in COL8A2. Guttae appeared coarser, more distinct, and elevated. Coalescence of guttae is visible (inset, near center) and clear areas of normal endothelial cells are interspersed.

1p35-p33.1. This had a maximum LOD score of 3.65 at marker D1S255, located $1 \mathrm{cM}$ from the COL8A2 gene, which was screened for mutations. This revealed a transversion mutation that changed leucine to tryptophan (TTG to TGG) at residue 450 , located in the collagen repeat domain of the COL8A2 protein. In this family, the mutant allele was always associated with the disease phenotype and was not found in unaffected relatives or spouses. The allele was not found in 112 background-matched, normal, white control subjects (224 chromosomes). None of the four previously reported ${ }^{14}$ pathogenic mutations of COL8A2 (Arg155Gln, Arg304Gln, Arg434His, and Gln455Lys) were found in probands from each of the 62 families with late-onset disease.

\section{Corneal Slit Lamp Photography and Confocal Specular Microscopy of Fuchs Families with COL8A2 and Non-COL8A2 Mutations}

Retroillumination of the cornea in representative patients with the COL8A2 mutation revealed a fine, patchy distribution of guttae in contrast to patients with late-onset FCD, in whom the guttae were more coarse and distinct. Areas of coalescence were evident with intervening nonaffected areas (Fig. 2A, 2B). Confocal specular microscopy of the endothelium in patients with the L450W-COL8A2 mutation demonstrated mildly elevated guttae that were associated with individual endothelial cells (Fig. 3A). One patient with the L450W-COL8A2 mutation exhibited endothelial anomalies characteristic of posterior polymorphous dystrophy (Fig. 4). In contrast, familial late onset FCD patients had typical guttae, which were sharply raised and typically located along the borders between endothelial cells (Fig. 3B).

\section{Age-Severity Profile of Families with FCD}

Figure 5 displays the association between age and severity among affected individuals. The average age of severely affected patients, those of grade 3 or greater, was roughly 40 years younger for the COL8A2 pedigree (Fig. 5, filled symbols) than for patients in the remaining 62 families. Comparing the ages of patients of grade 3 and higher from the currently examined affected members of the Magovern pedigree (without parentheses) with the ages of other familial cases in the same severity range, we can establish the significance of early onset with a high level of confidence $\left(P=5 \times 10^{-4}\right) .^{25}$

The COL8A2 pedigree, in which some patients were reexamined after 25 to 29 years, also provides direct data on progression. Figure 5 represents two girls at severity grade 1,
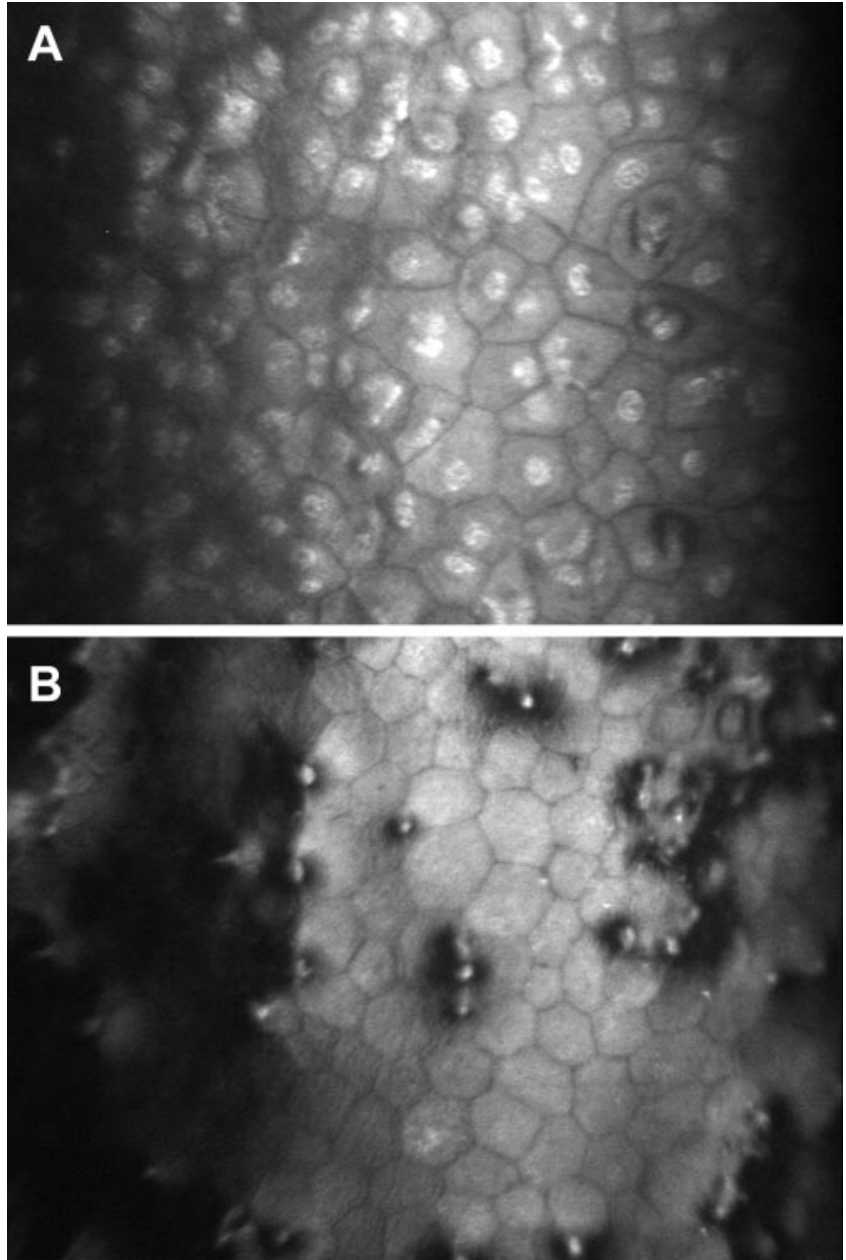

Figure 3. (A) Confocal specular microscopy of the endothelium of a representative grade 3 patient (age 32) with the L450W-COL8A2 mutation (see Fig. 1). Every endothelial cell in this image was associated with a single, low-elevation gutta, a pattern of high density that alternated with large areas devoid of guttae. (B) A patient with late-onset grade 3 FCD, without known COL8A2 mutations. Guttae were more typical of the disease: sharply raised, with higher elevation, and showing some areas of coalescence (top right). Areas of lower density were common, in which most endothelial cells were found without associated guttae. 


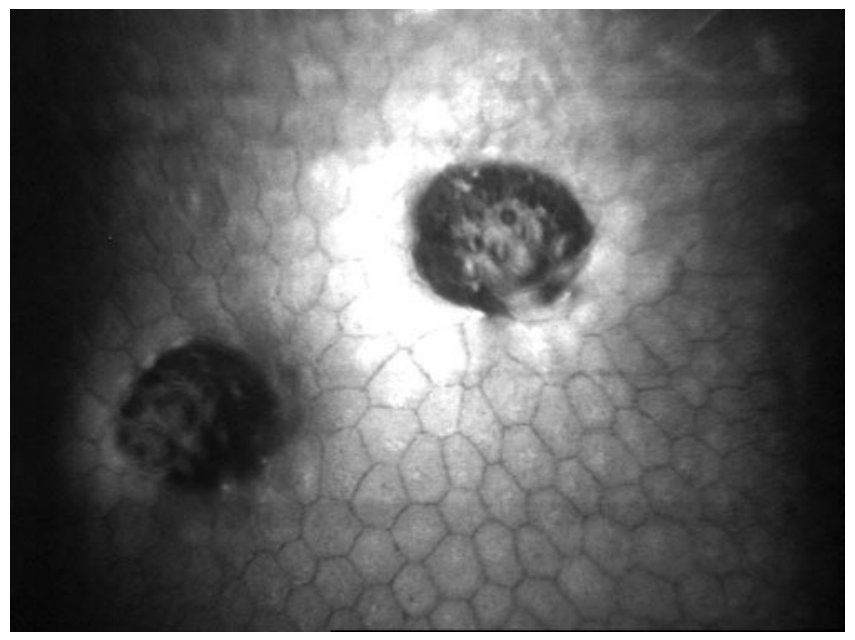

Figure 4. Confocal microscopy of a patient (age 10) with the COL8A2- L450W mutation (Fig. 1, hatched arrow). This was the only patient in the pedigree with this atypical phenotype. The large mulberry-like focal excrescences in the corneal endothelium are characteristic of PPCD. COL8A2-type guttae were present in the surrounding, more normal endothelium.

who were aged 3 and 4 years (Fig. 1; filled circles with brackets, open and filled arrows in Fig. 1) when examined in 1974, and progressed to grades 4 and 5 over the next 29 years. The dotted line connecting their 1974 and 2003 symbols gives a minimal increase of one severity grade every 8 years. Disease progression in these two individuals proceeded at a rate that closely follows the age versus severity distribution of the static data points, roughly 1 grade unit per 5 years (Fig. 5, filled symbols).

For the more heterogeneous patients with late-onset FCD (Fig. 5, open symbols) graphing the mean ages for each severity group indicates that the average age of the patients advances 5 years as one moves up each severity grade. This is surprisingly similar to the age-versus-severity relationship observed in the patients with early-onset disease bearing L450WCOL8A2, although median age of onset for this group is at least 40 years later. Another interesting feature of the graph is that the mean age of each severity grade follows a linear progression for both early and late disease. The definitions of the standard severity grading system of Krachmer et al. ${ }^{9}$ may have been selected to give a similar fraction of the total patient population in each grade, which would have normalized the results toward a linear profile. Finally, it should be noted that the two open symbols at ages 10 and 13 years are members of a single large kindred segregating late-onset FCD. They represent a special case, in that for each child, both parents were affected with late-onset FCD, and the morphology of their guttae was typical of late-onset disease.

\section{Sex Ratios of Patients with FCD}

Table 1 compares the sex distribution within the COL8A2 pedigree with that of 62 other familial FCD pedigrees. Both affected individuals and total members of the COL8A2 pedigree approximated a 1:1 female-male ratio, whereas affected individuals in the late-onset pedigrees were approximately $2.5: 1$. This excess of females in the 62 other pedigrees cannot be explained by chance variations of an underlying $1: 1$ ratio $(P<0.01)$ and confirms earlier findings for the general patient population. ${ }^{2,9}$ Conversely, the observed female-male ratio of 12:14 among affected members of the L450W-COL8A2 pedigree is very unlikely to fit a $2.5: 1$ ratio $(P<0.01)$, or in a $2: 1$ ratio $(P<0.03)$.

\section{Discussion}

In a large family with early-onset $\mathrm{FCD},{ }^{12}$ we have identified a mutation in the gene that encodes the $\alpha-2$ chain of collagen VIII, a major component of Descemet's membrane. This COL8A2 point mutation, $\mathrm{L} 450 \mathrm{~W}$, segregates with the disease as a dominant mutant at $100 \%$ penetrance. Our finding is of importance because this is only the second linkage-confirmed mutation reported for FCD. We also discovered that patients in this pedigree are very distinct from those with common forms of FCD in both morphologic features and temporal progression of the disease and suggest that this and the previously reported Q455K-COL8A2 mutations are associated with a rare earlyonset subtype of FCD. Although the COL8A2 variants R155Q, $\mathrm{R} 304 \mathrm{Q}$, and R434H were originally found in late-onset FCD and have been presented as evidence that this gene is also involved in common forms of the disease,${ }^{14}$ recent evidence indicates that R155Q is found at the same frequency in normal control subjects and is therefore unlikely to be pathogenic. ${ }^{16}$ There have been no further reports that implicate R304Q or R434H in FCD, and the pathogenicity of these variants is also unconfirmed. Without strong linkage data or evidence of defects in
FiguRE 5. Graph of data from single patient examinations, plotting the severity grade of Fuchs dystrophy versus age. All subjects shown had a severity grade of at least 1 , indicating that they were clearly affected. Some symbols were adjusted slightly up or down to show each data point. Squares: males, circles: females. Filled symbols: individuals from the $L 540 W$-COL8A2 pedigree. Filled symbols in brackets: Krachmer grades 9 derived from examination results used by Magovern et al. ${ }^{12}$ Solid symbols inside circles represent age and severity at the time of corneal transplantation (which ends the disease course). For the Magovern pedigree, actual disease progression is indicated for two females, who initially both had severity grade 1 in 1974, when they were examined at ages 3 and 4 years. Their data are connected by a dotted line to a point representing the mean age and severity.

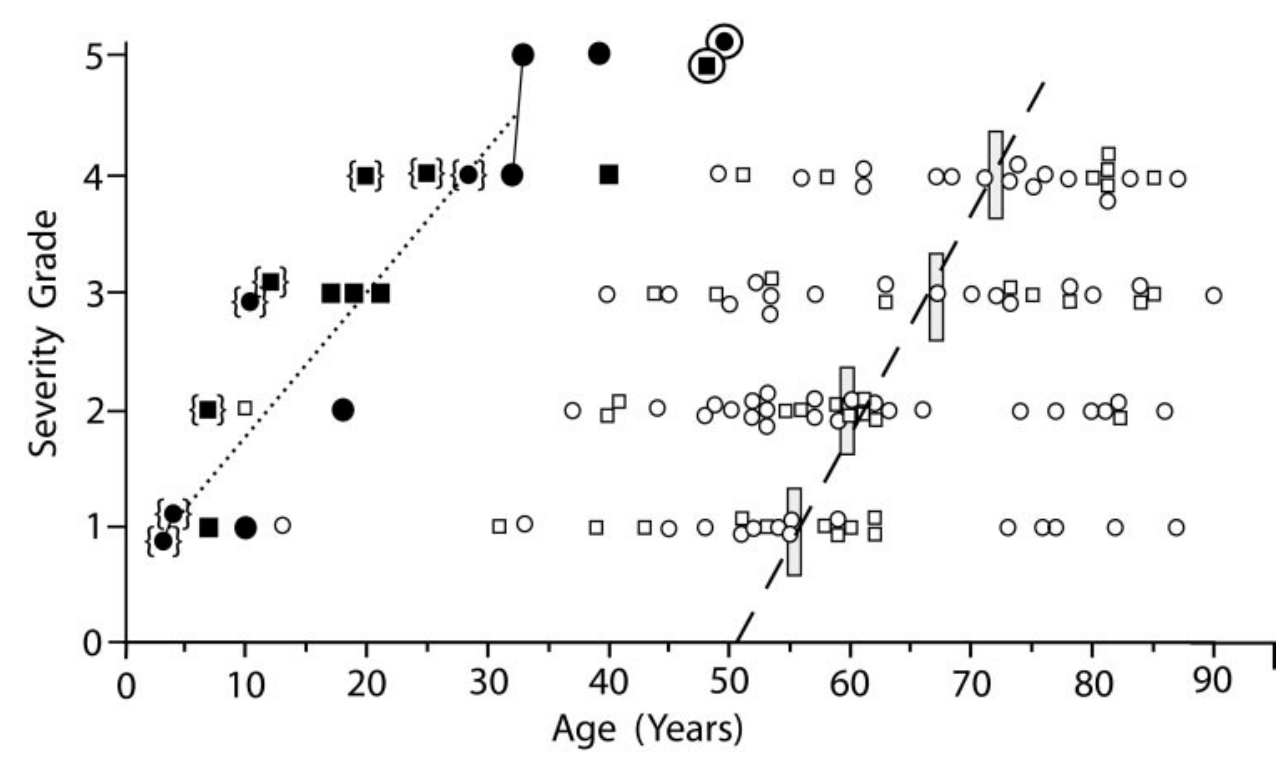


TABle 1. Number of Each Sex in the COL8A2- Pedigree and the Remaining 62-Late-Onset Pedigrees

\begin{tabular}{|c|c|c|c|c|}
\hline & \multicolumn{2}{|c|}{ COL8A2 Pedigree } & \multicolumn{2}{|c|}{ Other Pedigrees } \\
\hline & Female & Male & Female & Male \\
\hline Affected & 12 & 14 & 143 & 58 \\
\hline Normal+unknown & 16 & 15 & 104 & 103 \\
\hline TOTAL & 28 & 29 & 247 & 161 \\
\hline
\end{tabular}

All male and female affected individuals in the full COL8A2 pedigree (Fig. 1), including those examined in 2003 and 1979, were counted. Below these figures are the number of males and females remaining in the pedigree, including those known to be unaffected, combined with a smaller number of individuals of unknown disease status. The two right-hand columns represent the same categories for all 62 pedigrees that showed evidence of familial inheritance of lateonset FCD.

biochemical function, it not easy to make a case that amino acid substitutions cause a disease, ${ }^{26}$ as benign variants are the most common and can vary widely in frequency between different populations. ${ }^{27}$

\section{Morphologic Features}

We have shown that guttae in the L450W-COL8A2-carrying patients have a structure and distribution that differs considerably from that of patients with common late-onset FCD. ${ }^{5,6}$ Although there was no discussion of the morphology of guttae in the earlier report on the Q455K-COL8A2 mutation, it presented a confocal micrograph (see Fig. 3B of Reference 14) of guttae with morphology strikingly similar to those in the L450W-COL8A2-bearing patients. The Q455K-COL8A2 patient also had small, shallow guttae positioned near the center of the endothelial cells. It is of interest whether these features are a general feature of FCD-causing mutations in COL8A2. The mechanism by which COL8A2 mutations lead to the formation and positioning of guttae may be slightly different from that in late-onset disease, in which the guttae generally appear first at cell-cell junctions, near the basolateral face of the endothelial cells.

Another finding shared between the L450W and Q455KCOL8A2 mutations is the sporadic diagnosis of PPCD (OMIM 122000). This condition involves the development of vesicles with the epithelial transformation of endothelial cells. It is usually not progressive but can be accompanied by corneal decompensation. In a rare hereditary form of PPCD that has been mapped to $20 \mathrm{q} 11$, endothelial cell proliferation can occlude aqueous outflow. ${ }^{28}$ FCD is distinguished from PPCD by the fact that it lacks these vesicles and the endothelium retains its histologic character. ${ }^{4}$ In one 12 -year-old L450W-COL8A2 patient with PPCD, we found highly characteristic mulberrylike vesicles. There were no signs of guttae outside the vesicles, which supports the interpretation that this may be an alternate disease course. It is possible that this single patient in the pedigree had genetic or environmental modifying factors.

\section{Early-Onset and Homogeneity of Disease Progression}

With the L450W-COL8A2 mutation, children as young as 3 years have been found to be affected. All who were in the early stages of the disease at the time of the 1979 study have since progressed to corneal decompensation, and several have undergone penetrating keratoplasty. This natural history fits well with earlier reports of the Q455K-COLA2 mutation, in which FCD was diagnosed in individuals from ages 21 to 48 , and those in their 30s and 40 s had advanced stages of disease. Because no member of the Q455K family appears to have been screened for very early stages of the disease, it cannot be ruled out that some were affected as early as age 3 .

A unique feature of the L $450 \mathrm{~W}$ family is that we have been able to combine data collected in the 1970 s with recent examinations of the same individuals, as well as to ascertain new patients. In some cases, disease severity was graded at the time of corneal transplantation. The age-versus-severity distribution of the L450W-COL8A2-carrying patients is much narrower than that of the other 201 patients with late-onset disease. This is not unexpected, because the late-onset group very likely involved great heterogeneity, both in the genes involved and the severity of the specific mutations.

\section{The Sex Ratio}

In the L450W-COL8A2 family, the ratio of females to males was roughly $1: 1$, as originally noted in 1979 . What is new is that there are now enough individuals to show that, statistically, this number is not compatible with the $2: 1$ or $3: 1$ female-male ratios long observed for clinical cases of FCD. For the broader patient population we confirm earlier findings ${ }^{2,9}$ of a $2.5: 1$ female-male ratio for a group of 201 familial patients with late-onset FCD. What is clear is that the Mendelian COL8A2 mutation follows conventional expectations of a 1:1 ratio, and does not conform to the peculiar 2.5:1 ratio of common FCD. Among those older than 50 years, the greater longevity of women is expected to give a female-male ratio that is between 1.2:1 and 1.3:1, a range that is insufficient to explain to explain the 2.5:1 distribution in FCD.

\section{Disease Mechanisms}

How mutations in the COL8A2 gene cause the clinical and histopathological features of FCD or PPCD remains unknown. Collagen VIII is a short-chain, nonfibrillar, extracellular matrix component with a composition that varies between tissues $^{29,30}$ and there is evidence that it may serve a structural role $^{31}$ or be involved in cellular differentiation. ${ }^{30}$ In FCD, it appears to accumulate at higher levels within Descemet's membrane in the posterior collagenous zone, ${ }^{31,32}$ which appears to contribute structurally to the guttae. The $\alpha 1$ and $\alpha 2$ chains of collagen VIII assemble in vivo as trimers by their collagen helix domains, and form a hexagonal lattice. ${ }^{33}$ The subunit composition of these trimers within the lattice remains unknown, as does the relationship of the lattice structure itself to the biological functions of Descemet's membrane. ${ }^{34,35} \mathrm{Be}$ cause collagen VIII is also produced by vascular endothelial cells, ${ }^{30}$ one would expect a mutation that destroyed the basic functions of the COL8A2 protein to have severe and systemic effects that were not seen in these patients with FCD in our study. Our discovery of a second FCD-related dominant mutation affecting a small, conserved interval of the COL8A2 collagen helix domain suggests that this region may be of selective importance to the function of this protein in the cornea, as these mutations have no obvious effects elsewhere in the body.

We suggest two very different disease mechanisms. First, genetically abnormal collagen VIII may interfere with cell adhesion and differentiation of the endothelium, as in PPCD. In $\mathrm{FCD}$, it is possible that mutant COL8A2 proteins improperly interact with other molecules in the extracellular matrix, resulting in sites of structural weakness in Descemet's membrane ${ }^{34,35}$ that allow the extrusion of material that forms the guttae. The irregular basal lamina topography appears to indent physically and stretch the endothelial cells, ${ }^{3,8}$ which may compromise their ability to transport electrolytes and maintain corneal clarity.

\section{References}

1. Mohamadi P, McDonnell JM, Irvine JA, McDonnell PJ, Rao N, Smith RE. Changing indications for penetrating keratoplasty, 1984-1988. Am J Opbthalmol. 1989;15:550-552. 
2. Fuchs E, Dystrophia epithelialis corneae. Graefes Arch Clin Exp Ophthalmol. 1910;76:478-508.

3. Wilson SE, Bourne WM. Fuchs' dystrophy. Cornea. 1988;7:2-18.

4. Waring GO, Bourne WM, Edelhauser HF, Kenyon KR. The corneal endothelium, normal and pathologic structure and function. $O p b$ thalmology. 1982;89:531-590.

5. Jackson A, Robinson F, Frazer D, Archer D. Corneal guttata: a comparative clinical and specular micrographic study. Eye. 1999; 13:737-743.

6. Chiou AG, Kaufman SC, Beuerman RW, Ohta T, Soliman H, Kaufman HE. Confocal microscopy in cornea guttata and Fuchs' endothelial dystrophy. Br J Ophthalmol. 1999;83:185-189.

7. Li QJ, Ashraf MF, Shen DF, et al. The role of apoptosis in the pathogenesis of Fuchs endothelial dystrophy of the cornea. Arch Ophthalmol. 2001;119:1597-604.

8. Bergmanson JP, Sheldon TM, Goosey JD. Fuchs' endothelial dystrophy: a fresh look at an aging disease. Ophthalmic Physiol Opt. 1999;19:210-222.

9. Krachmer JH, Purcell JJ Jr, Young CW, Bucher KD. Corneal endothelial dystrophy: a study of 64 families. Arch Ophthalmol. 1978; 96:2036-2039.

10. Cross HE, Maumenee AE, Cantolino SJ. Inheritance of Fuchs' endothelial dystrophy. Arch Ophthalmol. 1971;85:268-272.

11. Rosenblum, P, Stark, WJ, Maumenee IH, Hirst LW, Maumenee AE. Hereditary Fuchs' dystrophy. Am J Ophthalmol. 1980;90:455462.

12. Magovern M, Beauchamp B, McTigue JW, Baumiller RC. Inheritance of Fuchs' combined dystrophy. Ophthalmology. 1979;86: 1897-1923.

13. Chang SW, Tuli S, Azar D. Corneal dystrophies. In: Traboulsi E, ed. Genetic Diseases of the Eye: a Textbook and Atlas. New York: Oxford University Press; 1998;Chap 13.

14. Biswas S, Munier FL, Yardley J, et al. Missense mutations in COL8A2, the gene encoding the $\alpha 2$ chain of type VIII collagen, cause two forms of corneal endothelial dystrophy. Hum Mol Genet. 2001;10:2415-2423.

15. Klintworth GK. The molecular genetics of the corneal dystrophies: current status. Front Biosci. 2003;8:687-713.

16. Kobayashi A, Fujiki K, Murakami A, et al. Analysis of COL8A2 gene mutation in Japanese patients with Fuchs' endothelial dystrophy and posterior polymorphous dystrophy. Jpn J Ophthalmol. 2004; 48:195-198.

17. Bigar F. Specular microscopy of the corneal endothelium. Optical solutions and clinical results. Dev Ophthalmol. 1982;6:1-94.

18. Boehnke M, Cox NJ. Accurate inference of relationships in sib-pair linkage studies. Am J Hum Genet. 1997;61:423- 429.
19. Broman KW, Weber JL. Estimation of pairwise relationships in the presence of genotyping errors. Am Hum Genet. 1998;63:1563-1564.

20. O'Connell JR, Weeks DE. PedCheck: a program for identification of genotype incompatibilities in linkage analysis. Am Hum Genet. 1998;6:259-266.

21. Broman KW. Estimation of allele frequencies with data on sibships. Genet Epidemiol. 2001;20:307-315.

22. Sobel E, Lange K. Descent graphs in pedigree analysis: applications to haplotyping, location scores, and marker sharing statistics. $\mathrm{Am}$ Hum Genet. 1996;58:1323-1337.

23. Broman KW, Murray JC, Sheffield VC, White RL, Weber JL. Comprehensive human genetic maps: individual and sex-specific variation in recombination. Am Hum Genet. 1998;63:861-869.

24. Dracopoli NC, ed. Current Protocols in Human Genetics. New York: Current Protocols; 1994.

25. Manly BFJ. Randomization, Bootstrap and Monte Carlo Methods in Biology. 2nd ed. London: Chapman \& Hall; 1997.

26. Kazazian HH, Antonarakis SE. The varieties of mutation. Prog Med Genet. 1988;7:43-67.

27. Kidd KK, Pakstis AJ, Speed WC, Kidd JR. Understanding human DNA sequence variation. $J$ Hered. 2004;95:406-420.

28. Heon E, Mathers WD, Alward WL, et al. Linkage of posterior polymorphous corneal dystrophy to 20q11. Hum Mol Genet. $1995 ; 4: 485-488$.

29. Muragaki Y, Jacenko O, Apte S, Mattei MG, Ninomiya Y, Olsen BR. The $\alpha 2$ (VIII) collagen gene: a novel member of the short chain collagen family located on the human chromosome 1.J Biol Chem. 1991;266:7721-7727.

30. Sage H, Iruela-Arispe ML. Type VIII collagen in murine development: association with capillary formation in vitro. Ann NY Acad Sci. 1990;580:17-31.

31. Levy SG, Moss J, Sawada H, Dopping-Hepenstal PJ, McCartney AC. The composition of wide-spaced collagen in normal and diseased Descemet's membrane. Curr Eye Res. 1996;15:45-52.

32. Kenney MC, Labermeier U, Hinds D, Waring GO III. Characterization of the Descemet's membrane/posterior collagenous layer isolated from Fuchs' endothelial dystrophy corneas. Exp Eye Res. 1984;39:267-277.

33. Greenhill NS, Ruger BM, Hasan Q, Davis PF. The $\alpha 1$ (VIII) and $\alpha 2$ (VIII) collagen chains form two distinct homotrimeric proteins in vivo. Matrix Biol. 2000;19:19-28.

34. Illidge C, Kielty C, Shuttleworth A. Type VIII collagen: heterotrimeric chain association. Int J Biochem Cell Biol. 2001;33:521529.

35. Stephan S, Sherratt MJ, Hodson N, Shuttleworth A, Kielty CM. Expression and superamolecular assembly of recombinant $\alpha 1$ (VIII) and $\alpha 2$ (VIII) collagen homotrimers. J Biol Chem. 2004;279: 21469-21477. 